\title{
An Evaluation of Reinforcer Magnitude and Echoic Prompts on Vocal Requesting of Individuals with Autism Spectrum Disorder
}

\author{
Reem Muharib ${ }^{1}$ (D) Russell Lang ${ }^{1} \cdot$ Virginia L. Walker ${ }^{2} \cdot$ Andrew Phinney $^{3}$. \\ Marcela Rodriguez ${ }^{3}$
}

Accepted: 27 January 2021 / Published online: 10 February 2021

(C) The Author(s), under exclusive licence to Springer Science+Business Media, LLC part of Springer Nature 2021

\begin{abstract}
Individuals with autism spectrum disorder (ASD) often have communication support needs and many rely on augmentative and alternative communication (AAC) systems to communicate. Previous research suggests that AAC use does not preclude the acquisition of spoken language and, in some cases, may facilitate improvements in spoken communication in children with ASD. This study systematically applied echoic prompting and manipulated reinforcer magnitude in an effort to increase the spoken requests of 3 children with ASD, ages 10-12 years, who used iPad-based speech generating devices (SGDs). The spoken language (vocal requests) of all 3 participants increased in frequency and 1 participant began using spoken language exclusively, even when the SGD was an option. Implications for practitioners and directions for future research in this area are discussed.
\end{abstract}

Keywords Autism spectrum disorder - Augmentative and alternative communication $\cdot$ Reinforcer magnitude $\cdot$ Speech-generating devices $\cdot$ Vocalizations

Individuals with autism spectrum disorder (ASD) have social communication support needs, and as many as $31 \%$ may not produce spoken language at all (Newman et al. 2011; Wodka et al. 2013). Augmentative and alternative communication (AAC) systems have been found to be effective supplements for individuals with spoken language deficits and effective replacement communication modalities for those with a complete absence of functional speech. There are many evidence-based

Reem Muharib

r_m806@txstate.edu

1 Texas State University \& Clinic for Autism Research Evaluation and Support, 601 University Blvd, San Marcos, TX 78666, USA

2 University of North Carolina at Charlotte, Charlotte, NC, USA

3 Texas State University, San Marcos, TX, USA 
AAC options that generally can be classified as either aided-AAC (i.e., systems that require equipment such as pictures cards or a portable electronic device) or unaidedAAC systems (i.e., systems that do not require equipment such as hand signs). Recently, in terms of aided-AAC, there has been an increase in the use of portable electronic devices (e.g., iPads) to house software capable of rendering the iPad into a speech generating device (SGD). Prior to the term SGD, devices with similar functionality (i.e., push-button speech production devices) were referred to as voice output communication aides (VOCA). The combined research literature involving VOCAs and SGDs is sufficient to classify this approach as evidence-based practice (Muharib and Alzrayer 2018).

One potential limitation to the use of AAC is the concern that reliance on such devices may preclude the user from eventually acquiring speech (Beukelman and Mirenda 2013). Specifically, if AAC use fulfills communication needs, then opportunities and motivation to develop speech may decrease precluding acquisition of spoken language. However, previous research has measured spoken language before, during, and after AAC use, and the best evidence suggests that AAC use does not inhibit the development of speech among individuals with developmental disabilities (e.g., Millar 2009; Millar et al. 2006; Schlosser et al. 2009; Schlosser and Wendt 2008). Further, there is evidence that AAC use may actually facilitate the development of speech in some children (Blischak et al. 2003; Kasari et al. 2014; Schlosser et al. 2007).

There are several reasons why increasing functional vocalizations (e.g., vocal requests) may be a socially valid intervention goal for children with ASD who use AAC. First, given the possibility that SGDs may become inoperable or inaccessible at unanticipated times (e.g., loss of battery power, theft, and breakage), children who rely on SGDs would benefit from acquiring multiple communication strategies to avoid and repair communication breakdowns (Cagliani et al. 2019; Gevarter et al. 2016). Second, transfer to alternative forms of communication may be necessary for some individuals due to changing conditions (e.g., deteriorating visual acuity). Third, if stakeholders believe adopting SGDs for communication means abandoning hope of developing spoken language (Beukelman and Mirenda 2013; Schlosser and Wendt 2008), they may hesitate to initiate SGD use and delay the child's acquisition of effective communication. Finally, developing speech may provide individuals with ASD an opportunity to communicate with a wider range of communicative partners in a wider range of environments (Paul et al. 2013).

Previous research has evaluated an array of behavioral intervention components and teaching strategies in an effort to design AAC intervention protocols that may increase the probability of speech emerging in children with ASD (Lorah et al. 2013; Lorah and Parnell 2017). Ostensibly, intervention protocols designed to (a) provide additional opportunities for children to attempt speech, (b) increase the amount of effort necessary for the child to use AAC, (c) model speech, and (d) differentially reinforce speech over AAC appear to occasion an increase in the spoken language of some AAC users. For example, Cagliani et al. (2017) added a delay between participant requests (mands) using a Picture Exchange Communication System (Bondy and Frost 1994) and the delivery of the requested reinforcer. Three of the four participants began making vocal requests when the delay to reinforcement following 
picture exchange requests reached $5 \mathrm{~s}$. Next, a response-effort manipulation procedure was added that involved moving materials necessary for picture-based communication out of the participants' immediate reach to increase the effort required for picture exchange. The combination of reinforcer delay and increased response effort further increased the spoken requests of the three children who showed improvement following the reinforcer-delay alone. Similar results were obtained in a replication study also by Cagliani et al. (2019) wherein reinforcer delay and response effort manipulation increased speech in two of three more children with ASD, ages 8 to 9 years, who used aided-AAC (i.e., SGD and picture exchange).

Echoic prompting has also been used to increase spoken language in children who use aided-AAC. For example, Alzrayer et al. (2020) taught three children (ages 5 to 8 years) with developmental disabilities to request items using an iPad-based SGD. Echoic prompts (e.g., "say chips") were delivered by therapists immediately after the child used their SGD to request. Although spoken language increased, the effect was inconsistent. However, when the SGD was removed, the spoken requests of all three children improved to mastery criteria. The potential for echoic prompting to improve spoken language of children with ASD who use AAC was also demonstrated by Carbone et al. (2010) who reported the emergence of speech in children with minimal vocal repertoire who used manual sign. Specifically, Carbone et al. implemented a 5-s prompt delay after a participant requested an item using a manual sign before providing an echoic prompt. Prompted vocalizations increased for all three children; however, two children showed minimal to no increase in unprompted vocalizations necessitating more research in this area.

Although the corpus of literature in this area is overall promising, not all previous research has reported positive findings. In particular, interventions consisting of only one intervention component (e.g., only a reinforcer delay or only echoic prompting but not both) have produced fewer desirable outcomes or no increase in spoken language (e.g., Bishop et al. 2020; Cagliani et al. 2017, 2019; Gevarter et al. 2016; Gevarter and Horan, 2018; Greenberg et al. 2014). For example, Greenberg et al. (2014) taught four children with developmental disabilities, ages 4 to 8 years, to request preferred items using picture exchange and spoken language. A 5-s time delay was implemented and contingent access to a reinforcer was given upon a picture exchange and/or vocal request. None of the participants showed increased vocal requests. In a second phase that included two participants, one participant showed increased vocal requests when the interventionist implemented a vocal model (e.g., "I want toy") as the child was receiving a reinforcer while the other required echoic prompts (i.e., prompting vocal requests before giving a reinforcer). While this study showed promising results, only two children showed an increase in vocal requesting and each required an individualized procedure precluding the generalizability of the results. Similarly, Bishop et al. (2020) implemented a 5-s reinforcer delay and differentially reinforced vocal requests of three children with ASD, ages 5 to 10 years, who had used SGDs. Vocal requests did not emerge with any of the three children. In a subsequent phase, Bishop et al. added echoic prompts which resulted in an increase of vocal requests for two of the three children. These previous studies showed variable vocal responding and demonstrated the need for future research 
using multi-component interventions aimed at increasing speech of individuals with ASD.

This current study aimed to replicate and extend previous research by demonstrating an increase in spoken language in response to intervention involving (a) changes to reinforcer magnitude and (b) an echoic prompting procedure targeting spoken requests. In order to examine changes in response allocation across communication modalities, independent SGD requests and independent vocal requests were compared during sessions with the SGD available. To extend previous research on speech production following SGD use, data were also collected on independent vocal requests following intervention when the SGD was not available.

\section{Method}

\section{Participants}

Institutional Review Board approval and informed consent from legal guardians was obtained prior to initiating this study. Teachers and therapists that typically work with children with ASD were asked to refer potential participants. The first three referrals who met the following inclusion criteria participated: (a) had a confirmed diagnosis of ASD from an appropriately trained professional who was not otherwise involved in this study, (b) demonstrated ability to independently use a portable electronic device (e.g., iPad) as a SGD to request a minimum of 10 different items, and (c) demonstrated ability to produce simple monosyllabic vocal imitations (e.g., "me," "oh," "wow") when asked. Individuals who did not meet those criteria or engaged in echolalic speech were excluded.

Eric was a 10-year-old Caucasian male diagnosed with ASD and speech impairment. He received academic instruction in a self-contained classroom. The Cognitive Scale of the Developmental Profile- $3^{\text {rd }}$ edition (DP3; Alpern et al. 2007) placed Eric below the 0.1st percentile. Eric scored 7 on the Early Echoic Skills Assessment (EESA; Esch 2008) indicating a sufficient echoic repertoire for participation. Eric was able to request at least 10 items (e.g., "water," "restroom," and "snack") using his SGD. Eric had been using his SGD across various settings (e.g., school and home) for two years. He had been receiving language and speech therapy through the school system 30 min twice a week for the last 3 years and this therapy was not altered during the study. Eric did not have a history of receiving any other therapy.

Sam was a 10-year-old Caucasian male diagnosed with ASD and speech impairment. He received academic instruction in a self-contained classroom. Sam scored 104 on the Gilliam Autism Rating Scales, $2^{\text {nd }}$ edition (GARS-2; Gilliam 2006), supporting his diagnosis of ASD. Sam's primary caregiver and teacher both completed the Adaptive Behavior Assessment System, $3^{\text {rd }}$ edition (ABAS-3; Harrison and Oakland 2015), scoring Sam in the extremely low range with 59 and 52, respectively. Sam scored 78.5 on EESA and demonstrated his ability to request at least 10 different items using his SGD. Sam had been using his SGD in school settings for a year. He had been receiving language and speech therapy through the school system 
30 min twice a week for a year, and this therapy was not altered during the study. Sam did not have a history of receiving any other therapy.

Juan was a 13-year-old Latino male diagnosed with ASD, Down syndrome, hearing impairment, and visual impairment who received services in a university-based clinical setting. Due to co-morbid diagnoses and sensory impairments, standardized assessments were deemed inappropriate for Juan. However, parent and therapist report followed by direct observation were used to confirm his eligibility to participate. Juan could request approximately 20 items and was able to produce a few onesyllable word approximations (e.g., "dat" for "that") as well as simple reduplicated syllables (e.g., "da-da.”). Juan had used a SGD across various settings (e.g., home and clinic) and received applied behavior analysis (ABA) therapy in a universitybased clinic for 4 years. Juan's visual acuity was decreasing over time due to Keratoconus (i.e., progressive thinning of the cornea). His worsening visual impairment made it increasingly difficult for Juan to see the SGD icons and underscored the importance of developing his spoken language.

\section{Settings}

Trained interventionists conducted all sessions individually (one-to-one) with each participant. During sessions, the interventionist sat across from the participant at a table. Sam and Eric's sessions were conducted in unused rooms in their public elementary school and were implemented by their typical classroom teacher with 5 years of experience in special education. Juan's sessions were conducted in a university-based clinic room and were implemented by his typical behavioral therapist. The therapist had 2 years of experience delivering behavioral interventions to children with ASD in clinical settings. Both the teacher and therapist were receiving graduate training toward certification as behavior analysts (BCBA). Prior to the study, the first author provided the teacher and therapist (hereafter referred to as interventionists) with individual training on the study procedures using Behavioral Skills Training (i.e., instruction, rehearsal, role-play, and feedback; Brock et al. 2017) until they met the mastery criterion of $100 \%$.

\section{Materials}

Materials included iPads loaded with SGD software applications (app) and preferred items (reinforcers). The iPads and SGD software apps already being used by participants prior to the study were used in the study. In terms of SGD software, Eric's iPad was loaded with LAMP Words for Life ${ }^{\circledR}$, Sam used TouchChat ${ }$, and Juan used ProloQuo2go®. Preferred items were identified for each individual participant via a multiple stimulus without replacement preference assessment (MSWO; DeLeon and Iwata 1996). Selected items included bubbles, sprinklers, spinner, and pretzels for Eric; bubbles, spinner, and magnets for Sam; and cookies, chips and videos for Juan. 


\section{Dependent Measure and Data Collection}

Interventionists collected data on independent vocal requests and iPad requests on a trial-by-trial basis wherein each variable was scored as present or absent. An independent vocal request was scored when (a) the request occurred before the interventionist delivered the echoic prompt and (b) the request was an audible and intelligible vocal approximation that corresponded to one of the preferred objects placed in front of the participant (i.e., available reinforcers). Specific vocal approximations were operationally defined for each participant based on their individual preferred items and articulation skills. For Eric and Juan, a vocal request had to match at least one syllable of the reinforcer (e.g., "Tube" for YouTube, "pinner" for spinner, "teeto" for Cheetos). For Sam, a vocal response had to consist of a complete sentence (e.g., "Can I have spinner, please?"). Vocal responses were selected based on therapist's/teacher's input as well as EESA scores (for Sam and Eric).

During the first phase of intervention, when the SGD was available, independent SGD requests were also recorded to track changes in response allocation between vocal and SGD requests across baseline and intervention. An independent SGD request was defined as touching an icon on the iPad's display that corresponded to one of the available reinforcers with sufficient force for speech output.

\section{Interobserver Agreement}

All sessions were recorded to permit secondary coding and calculate interobserver agreement (IOA). Data collection sheets from 30\% of sessions for all phases and participants were randomly selected for IOA. IOA was calculated by comparing data collected by the interventionists to data extracted by the first author from the recordings using a trial-by-trial formula. Specifically, the number of agreements was divided by the total number of agreements and disagreements and multiplied by 100 (Kazdin 1982). For Eric, the IOA average was 99\% on vocal requests (range $=90-100 \%$ ) and $100 \%$ for SGD requests. For Sam and Juan, IOA was $100 \%$ on vocal requests and SGD requests.

\section{Research Design}

We used a multiple probe across participants design (Horner et al. 2005) to examine the effects of reinforcer magnitude and echoic prompting on vocal requests by three children who used SGDs to request preferred objects. All three participants began baseline at the same time and intervention implementation was staggered across participants. Before transitioning to the second phase of intervention, mastery criterion for the first phase required producing eight or more independent vocal requests across six consecutive sessions. Before moving to the maintenance phase, the mastery criterion for the second phase required producing eight or more independent vocal requests across five consecutive sessions. Each participant received one to two sessions a day, each consisted of 10 trials, for a total of four to five sessions per 
week. Each baseline and intervention session lasted approximately 5-7 min. Sessions would have been discontinued if a participant's behavior suggested they did not want to continue (i.e., getting out of seat, pushing away from table, or in some other way suggesting a desire to stop). However, none of these behaviors occurred during sessions with any of the participants.

\section{Procedures}

\section{Preference Assessments}

A two-part preference assessment was used to identify preferred items for each participant. First, teachers and therapists were asked to identify items that would be appropriate in their educational setting (classroom and clinic) and, based on their experience with each child, they believed had the potential to function as a reinforcer. The resulting items were used as stimuli during a MSWO preference assessment (DeLeon and Iwata 1996). Specifically, the items identified as potential reinforcers were placed in an array in front of a participant and the participant was asked to select one. After selection, participants utilized the selected item (i.e., watched a video clip, played with a toy, or consumed an edible) and the remaining items were removed. This procedure continued until items could be ranked by relative preference. The MSWO was conducted three times before baseline and again every two weeks in an effort to ensure highly preferred items were used throughout the study.

\section{Baseline}

During baseline, the interventionist placed three to four preferred items and the SGD in front of the participant and commenced the session by saying, "If you want something, you can ask for it." The interventionists waited $4 \mathrm{~s}$ for a request to occur. In baseline, both vocal requests and SGD requests resulted in the larger magnitude of reinforcer (i.e., four bites of an edible or $20 \mathrm{~s}$ of access to a video or toy).

\section{Intervention (with SGD)}

Intervention procedures were identical to baseline with two exceptions. First, when a participant did not vocalize within $4 \mathrm{~s}$ of activating the SGD, the interventionist provided an echoic prompt (e.g., "Say Oreo.") using a constant time delay approach (Ault et al. 1988). In the first five trials, a 0 s prompt delay was used. The prompt delay was increased to $4 \mathrm{~s}$ in the last five trials. After a single session with 5 independent vocal responses, the $4 \mathrm{~s}$ prompt delay was used for all trials in all subsequent sessions.

Second, during intervention, the amount of reinforcer delivered (reinforcer magnitude; Ferguson et al. 2019) was contingent on the type of request produced. Specifically, a low/small magnitude reinforcer (i.e., one bite of an edible or $5 \mathrm{~s}$ access to video or toy) was contingent on (a) prompted vocal requests and (b) all SGD requests (SGD use was never prompted). High/large magnitude reinforcers (i.e., four 
bites of an edible or $20 \mathrm{~s}$ access to video or toy) were delivered contingent on independent vocal requests produced either before an SGD request or within $4 \mathrm{~s}$ of the prompt delay following SGD activation. In an effort to isolate reinforcer magnitude, no additional reinforcers (e.g., praise) were provided for any request.

\section{Intervention (No SGD)}

As in prior phases, three to four preferred items were placed in front of the participant and the interventionist said, "If you want something, you can ask for it." If the participant did not vocalize a request within $4 \mathrm{~s}$, an echoic prompt was delivered (e.g., "Say Oreo."). If the participant responded to the echoic prompt with a vocal request, one bite of an edible or $5 \mathrm{~s}$ access to reinforcer was delivered (i.e., low/small magnitude contingency). However, if an independent vocal request was produced prior to the echoic prompt, four bites of an edible or $20 \mathrm{~s}$ access to video or toy was provided (i.e., high/large magnitude contingency). All other forms of potential reinforcement (e.g., praise) were held constant. If a vocal response occurred after an echoic prompt, the interventionist would have provided a distractor one-step direction (e.g., "Touch your nose" and then delivered one bite of an edible or $5 \mathrm{~s}$ of video or toy). However, this did not occur during the study. From an ethical standpoint, we established criteria for moving Eric back to the first phase (with SGD) because he consistently relied on the SGD in the majority of trials in the previous phase. These were (a) no vocal response within $4 \mathrm{~s}$ of an echoic prompt across three trials, or (b) grabbing items without engaging in a vocal request across three trials. However, none of this occurred.

\section{Maintenance}

Maintenance data were collected for Sam at 1 and 2 weeks after withdraw of intervention. In these probes, the interventionist presented three to four preferred items in front of the participant without the SGD present and ran procedures identical to the final intervention phase. Maintenance data could not be collected for Eric or Juan due to school closures and health concerns arising during the COVID-19 pandemic.

\section{Procedural Fidelity}

The first author assessed procedural fidelity by reviewing a minimum of $30 \%$ of recordings selected randomly from every phase from every participant. A procedural checklist specific to each phase (baseline to maintenance) was scored to evaluate the extent to which the interventionist implemented the procedures accurately (checklists available on request). Examples of the checklist items include (a) presenting three to four preferred items in front of the participant, (b) providing a vocal prompt in instances where a participant did not produce a vocal request within $4 \mathrm{~s}$, and (c) providing the requested item contingent upon vocal requests (small magnitude for prompted, and large magnitude for independent responses). Procedural fidelity was calculated by dividing the number of steps implemented correctly by the total 
number of steps and multiplying by 100 . On average, procedural fidelity was $97 \%$ (range $=80-100 \%)$ for baseline, $99 \%$ (range $=91.6 \%-100 \%$ ) for the first intervention phase, $99.5 \%$ (range $=99-100 \%$ ) for the second intervention phase, and $100 \%$ for maintenance.

\section{Results}

Figure 1 shows the number of independent vocal requests and SGD requests for Eric, Sam, and Juan, and Table 1 identifies the specific vocalizations that emerged for each participant. Data in Fig. 1 were first analyzed using visual analysis to identify changes in data paths' level, trend, variability, and overlap as well as the immediacy of changes following presentation of independent variables and the time of changes across participants (Ledford and Gast 2018). Next, visual analysis was supplemented by calculating an effect size estimate using Tau-U, a nonparametric method for analyzing single-case design data (Parker et al. 2011). Tau-U scores range from 0 to 1 and can be interpreted as follows: $<0.20$ : small change, $0.20-0.60$ : moderate change, $0.60-0.80$ : large change, and $>0.80$ : large to very large change (Vannest and Ninci 2015). Baseline data were compared to data from the first intervention phase (i.e., SGD Present) to calculate Tau-U for vocal requests. The weighted average of Tau-U for vocal requests across all participants was 0.91 , suggesting a large to very large effect.

\section{Eric}

During baseline, Eric produced only one vocal request in 40 trials across four sessions $(M=0.25$, range $0-1)$, relying almost entirely on his SGD to request preferred items. When intervention initiated, Eric's vocal requests began to increase to a mean of eight vocal responses (range $=1-10$ ) across intervention sessions. After some initial variability, SGD requests remained high throughout the intervention sessions with the SGD present. When the SGD was removed during the second intervention phase, vocal requests remained high $(M=9$, range 8-10). Eric's Tau-U score for vocal requests was 0.99 , suggesting a large to very large effect.

\section{Sam}

During baseline, Sam did not produce any vocal requests and consistently used his SGD to request preferred items. During the first two sessions of intervention, vocal requesting remained at zero and SGD requests remained at 10 . Beginning in intervention session 3, vocal requests increased and SGD requests decreased rapidly and maintained at improved levels for the remainder of the phase $(M=7$, range $=0-10)$. When the SGD was removed, Sam produced independent vocal requests during every trial in every session $(M=10)$. Independent vocal requests were maintained at one and two weeks after termination of the intervention $(M=10)$. Sam's Tau-U score for vocal requests was 0.75 , suggesting a medium to high effect. 


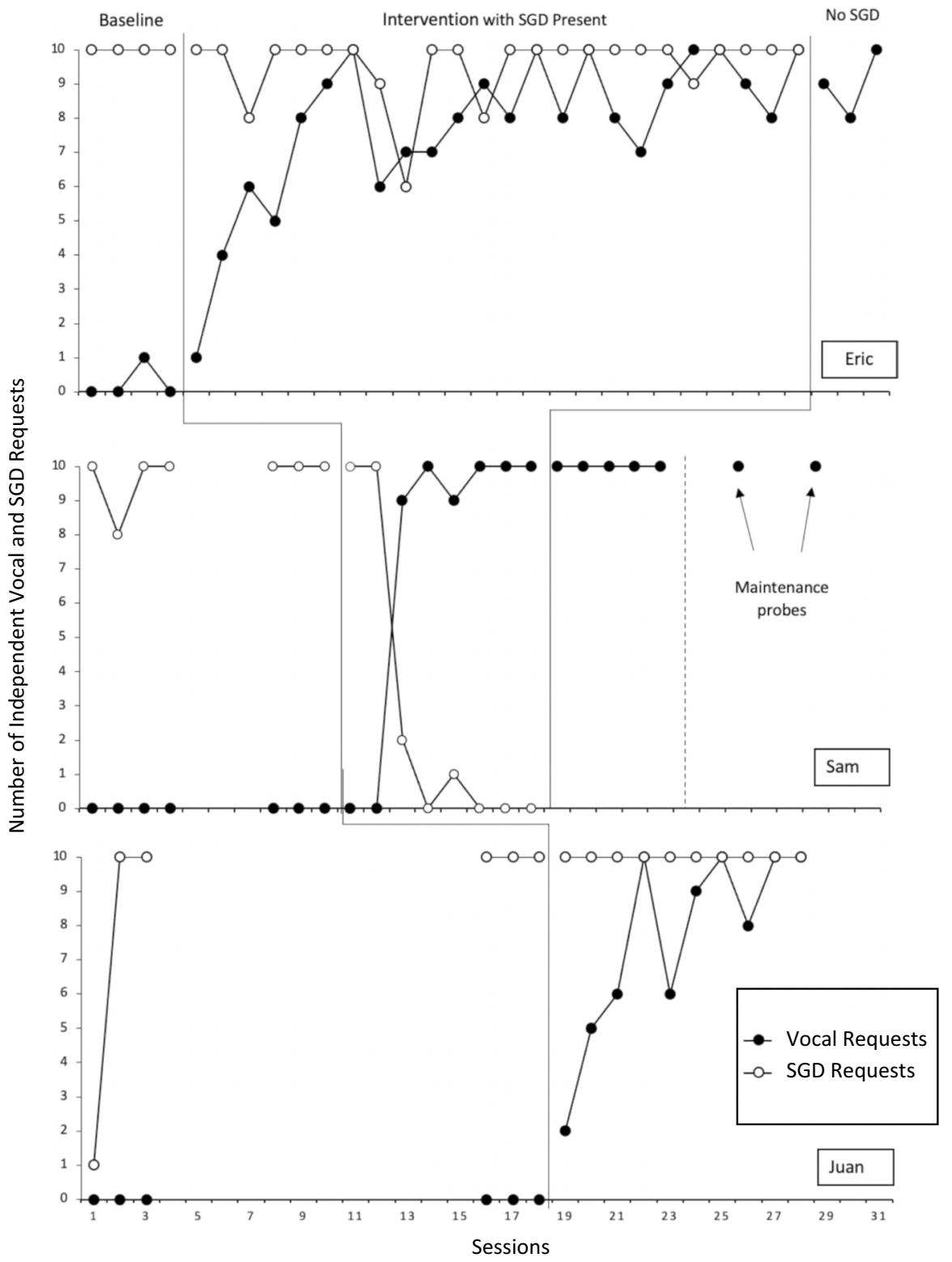

Fig. 1 Number of independent vocal and SGD requests

\section{Juan}

Juan did not produce any vocal requests during baseline and he consistently used his SGD to request preferred items. Intervention occasioned an immediate steady increase in vocal requests $(M=8$, range 2-8) and no change in SGD requests 
Table 1 Vocal approximations for preferred stimuli

\begin{tabular}{lll}
\hline Participants & Items Requested & Vocalizations \\
\hline Eric & Sprinkler & K-ah \\
& Bubbles & Ba-h, bubba-h \\
& Spinner & P-eh, pinner \\
Sam & Bubbles & Can I have bubbles, please? \\
& Spinner & Can I have spinner, please? \\
& Magnets & Can I have magnets, please? \\
\multirow{3}{*}{ Juan } & Oreo & Oo-yo \\
& Cheetos & Tee-tow \\
& YouTube & You-tu \\
\hline
\end{tabular}

$(M=10)$. Juan was not able to receive the second intervention due to school and clinic closures. Juan's Tau-U score for vocal requests was 1.0, which suggests a large effect.

\section{Discussion}

This study replicated previous research by demonstrating an increase in vocal requests in children with ASD who relied predominately on SGDs to communicate (e.g., Cagliani et al. 2017). Specifically, vocal requests were increased by echoic prompting and providing a larger magnitude of reinforcement (i.e., a larger quantity or longer duration) following vocal requests than provided following SGD requests. Visual analysis and Tau-U effect size estimates align to suggest that this approach resulted in large increases in vocal requesting for all three participants. The concern that SGD use may preclude the ability to further develop speech was not supported by this study (Beukelman and Mirenda 2013).

Several approaches to increasing spoken language for people who use AAC have been demonstrated in the literature and practitioners should consider several factors when designing an intervention for this purpose. As in most prior studies, this study used echoic prompting and arranged specific reinforcement contingencies. The property of reinforcement manipulated in this current approach was magnitude, which represents a procedural difference from previous research that has focused on the latency between a request and delayed reinforcer delivery in order to favor speech over picture exchange communication (e.g., Cagliani et al. 2019; Tincani et al. 2006). There are several factors practitioners should consider when determining which approach to use. For example, increasing reinforcer magnitude to favor speech would seem likely to reduce the probability of extinction effects relative to the delay to reinforcement strategy. Similarly, in cases involving challenging behavior maintained by access to preferred items, providing the lower quantity reinforcer following AAC use may result in lower levels of challenging behavior during sessions than a delay to reinforcement. Further, it would seem possible to embed manipulations of magnitude into a child's daily routine more easily than it would be 
to consistently implement delay to reinforcement in an applied setting. Finally, parents, individuals who AAC, and other stakeholders should be empowered to select between intervention components when multiple options are potentially effective.

In the first phase of intervention (SGD present), we compared the number of vocal requests to the number of SGD requests to consider how responses were allocated across the two communication options for each participant. One participant (Sam), who did not produce a single vocal request during baseline, stopped using the SGD and began exclusively making vocal requests after two intervention sessions. Although Eric and Juan produced zero to one vocal request in baseline, during intervention, they usually produced a SGD request before making a vocal request. Because they produced the vocal request following the SGD output but before receiving the echoic prompt from the interventionist, independent vocal requests increased while SGD use also remained consistently high. The reasons different patterns of response allocation between vocal and SGD requests may have emerged for Eric and Juan compared to Sam cannot be determined from this study because the research design did not control for potential influences. However, previous research suggests some factors that likely influenced these outcomes. For example, Sam had the most sophisticated echoic repertoire prior to participating in the study, which may have made vocal requesting less effortful than for Eric and Juan, who had more notable spoken language delays (Alzrayer et al. 2020; Johnston et al. 2004; Torelli et al. 2016). Similarly, Eric and Juan might have compensated for language deficits by relying on the SGD's output as a model for their vocalizations (Schlosser et al. 2007). Finally, it is also possible that Eric and Juan preferred using the SGD and were scrolling through their response class according to their individual preferences and history of reinforcement. Future research designed to address these possibilities may be helpful in continuing to develop intervention protocols in this area and to further elucidating the factors that contribute to beneficial outcomes of AAC.

The findings of this study should be considered in the context of a few limitations. First, we did not track the accuracy of Eric and Juan's vocal approximations over time. For example, we recorded "ba-h" and "bubba-h" as correct responses for "bubble" throughout the study, instead of measuring articulation. Future research should consider recording vocal approximations in more detail and attempt to identify changes in articulation and intelligibility in vocalizations over time. Second, generalization was not assessed and the extent to which increased vocalizations may extend to novel stimuli, environments, and communication partners should be considered in future studies. Relatedly, we implemented the intervention in a discrete trial format in empty school rooms and clinical settings. Embedding the intervention into the individual's natural routine may be more effective, efficient, and socially valid (Horner et al. 2005) and may ultimately facilitate generalized responding. Further, because we implemented vocal prompts and reinforcer magnitude manipulation simultaneously, it is unknown whether both components were necessary or whether one of the two components would have been sufficient to produce vocal requests. Future research could conduct a component analysis to consider the individual and combined effects of vocal prompts and reinforcer manipulation (e.g., components added sequentially with the sequence counter-balanced across participants) Additionally, given the range of results, diversity of language repertoire across 
participants, and the small sample size, the generalizability (external validity) of these results is a limitation and replications of these procedures with a larger sample of participants who have a similar language repertoire appear warranted. Finally, due to closures and safety concerns caused by the COVID-19 pandemic, we were unable to conduct the second intervention phase for Juan or collect maintenance data for Juan or Eric. Future research should collect maintenance data and consider telecommunication tools for data collection such as Zoom® in times in which schools and clinics are closed.

\section{Declarations}

Research Involving Human Participants and/or Animals All procedures performed in studies involving human participants were in accordance with the ethical standards of the institutional and/or national research committee and with the 1964 Helsinki Declaration and its later amendments or comparable ethical standards.

Informed Consent Informed consent was obtained from all individual participants included in the study.

Conflicts of Interest The authors declare that they have no conflicts of interest.

Ethical Approval This study was approved by the Institutional Review Board at Texas State University.

\section{References}

Alpern, G., Boll, T., \& Shearer, T. (2007). Developmental Profile (3rd ed.). Western Psychological Services.

Alzrayer, N. M., Muharib, R., \& Wood, C. L. (2020). Effects of a behavior intervention package on augmented and vocal mands by children with developmental disabilities. Journal of Developmental and Physical Disabilities, 32, 57-74. https://doi.org/10.1007/s10882-019-09681-5.

Ault, M. J., Gast, D. L., \& Wolery, M. (1988). Comparison of progressive and constant time-delay procedures in teaching community-sign word reading. American Journal of Mental Retardation, 93(1), 44-56.

Beukelman, D. R., \& Mirenda, P. (2013). Augmentative and alternative communication: Supporting children and adults with complex communication needs (4th ed.). Paul H. Brookes Publishing.

Bishop, S. K., Moore, J. W., Dart, E. H., Radley, K., Brewer, R., Barker, L. K., et al. (2020). Further investigation of increasing vocalizations of children with autism with a speech generating device. Journal of Applied Behavior Analysis, 53(1), 475-483. https://doi.org/10.1002/jaba.554.

Blischak, D. M., Lombardino, L. J., \& Dyson, A. T. (2003). Use of speech-generating devices: In support of natural speech. Augmentative and Alternative Communication, 19(1), 29-35. https://doi. org/10.1080/0743461032000056478.

Bondy, A., \& Frost, L. (1994). The picture exchange communication system. Focus on Autism and Other Developmental Disabilities, 9, 1-19.

Brock, M. E., Cannella-Malone, H. I., Seaman, R. L., Andzik, N. R., Schaefer, J. M., Page, E. J., et al. (2017). Findings across practitioner training studies in special education: A comprehensive review and meta-analysis. Exceptional Children, 84(1), 7-26. https://doi.org/10.1177/0014402917698008.

Cagliani, R., Ayres, K., Ringdahl, J., \& Whiteside, E. (2019). The effect of delay to reinforcement and response effort on response variability for individuals with autism spectrum disorder. Journal of Developmental and Physical Disabilities, 31, 55-71. https://doi.org/10.1007/s10882-018-9628-7. 
Cagliani, R., Ayres, R., Whiteside, K., \& Ringdahl, M. (2017). Picture exchange communication system and delay to reinforcement. Journal of Developmental and Physical Disabilities, 29, 925-939. https ://doi.org/10.1007/s10882-017-9564-y.

Carbone, V. J., Sweeney-Kerwin, E. J., Attanasio, V., \& Kasper, T. (2010). Increasing the vocal responses of children with autism and developmental disabilities using manual sign mand training and prompt delay. Journal of Applied Behavior Analysis, 43(4), 705-709. https://doi. org/10.1901/jaba.2010.43-705.

DeLeon, I. G., \& Iwata, B. A. (1996). Evaluation of a multiple-stimulus presentation format for assessing reinforcer preferences. Journal of Applied Behavior Analysis, 29(4), 519-533. https:// doi.org/10.1901/jaba.1996.29-519.

Esch, B. E. (2008). Early echoic skills assessment. In M. L. Sundberg (Ed.), Verbal behavior milestones assessment and placement program: The VB-MAPP (p. 24). AVB Press.

Ferguson, R. H., Falcomata, T. S., Ramirez-Cristoforo, A., \& Vargas Londono, F. (2019). An evaluation of the effects of varying magnitudes of reinforcement on variable responding exhibited by individuals with autism. Behavior Modification, 43(6), 774-789. https://doi.org/10.1177/01454 45519855615 .

Gevarter, C., \& Horan, K. (2018). A behavioral intervention package to increase vocalizations of individuals with autism during speech-generating device intervention. Journal of Behavioral Education, 28, 141-167. https://doi.org/10.1007/s10864-018-9300-4.

Gevarter, C., O’Reilly, M. F., Kuhn, M., Mills, K., Ferguson, R., Watkins, L., ..., Lancioni, G. E. (2016). Increasing the vocalizations of individuals with autism during intervention with a speech-generating device. Journal of Applied Behavior Analysis, 49(1), 17-33. https://doi. org/10.1002/jaba.270.

Gilliam, J. (2006). GARS-2: Gilliam Autism Rating Scale-Second Edition. PRO-ED.

Greenberg, A. L., Tomaino, M. E., \& Charlop, M. H. (2014). Adapting the picture exchange communication system to elicit vocalizations in children with autism. Journal of Developmental and Physical Disabilities, 26(1), 35-51. https://doi.org/10.1007/s10882-013-9344-2.

Harrison, P., \& Oakland, T. (2015). Adaptive Behavior Assessment System, Third Edition (ABAS-3). Western Psychological Services.

Horner, R., Carr, E., Halle, J., McGee, G., Odom, S., \& Wolery, M. (2005). The use of single subject research to identify evidence-based practice in special education. Exceptional Children, 71, $165-180$.

Johnston, S., Reichle, J., \& Evans, J. (2004). Supporting augmentative and alternative communication use by beginning communicators with severe disabilities. American Journal of Speech-Language Pathology, 13(1), 20-30. https://doi.org/10.1044/1058-0360(2004/004).

Kazdin, A. E. (1982). Single case research design: Methods for clinical and applied settings. Oxford: Oxford University Press.

Kasari, C., Kaiser, A., Goods, K., Nietfeld, J., Mathy, P., Landa, R., ... Almirall, D. (2014). Communication interventions for minimally verbal children with autism: A sequential multiple assignment randomized trial. Journal of the American Academy of Child \& Adolescent Psychiatry, 53(6), 635-646. https://doi.org/10.1016/j.jaac.2014.01.019.

Ledford, J. R., \& Gast, D. L. (2018). Single case research methodology (3rd ed.). Abingdon: Routledge.

Lorah, E., \& Parnell, A. (2017). Acquisition of tacting using a speech-generating device in group learning environments for preschoolers with autism. Journal of Developmental \& Physical Disabilities, 29, 597-609. https://doi.org/10.1007/s10882-017-9543-3.

Lorah, E., Tincani, M., Dodge, J., Gilroy, S., Hickey, A., \& Hantula, D. (2013). Evaluating picture exchange and the $\mathrm{iPad}^{\mathrm{TM}}$ as a speech generating device to teach communication to young children with autism. Journal of Developmental \& Physical Disabilities, 25(6), 637-649. https://doi. org/10.1007/s10882-013-9337-1.

Millar, D. C. (2009). Effects of AAC on natural speech development of individuals with autism spectrum disorders. In Mirenda, P., Iacono, T. (Eds.), Autism Spectrum Disorders and AAC (pp. 171-192). Paul H. Brookes Publishing Co.

Millar, D. C., Light, J. C., \& Schlosser, R. W. (2006). The impact of augmentative and alternative communication intervention on the speech production of individuals with developmental disabilities: A research review. Journal of Speech Language and Hearing Research, 49(2), 248-264. https://doi. org/10.1044/1092-4388(2006/021). 
Muharib, R., \& Alzrayer, N. M. (2018). The use of high-tech speech-generating devices as an evidencebased practice for children with autism spectrum disorders: A meta-analysis. Review Journal of Autism and Developmental Disorders, 5, 43-57. https://doi.org/10.1007/s40489-017-0122-4.

Newman, L., Wagner, M., Knokey, A.M., Marder, C., Nagle, K., Shaver, D., Wei, X., with Cameto, R., Contreras, E., Ferguson, K., Greene, S., \& Schwarting, M. (2011). The post-high school outcomes of young adults with disabilities up to 8 years after high school. A report from the National Longitudinal Transition Study-2 (NLTS2). (NCSER 2011-3005). Menlo Park: SRI International.

Parker, R. I., Vannest, K. J., Davis, J. L., \& Sauber, S. B. (2011). Combining nonoverlap and trend for single case research: Tau-U. Behavior Therapy, 42(2), 284-299. https://doi.org/10.1016/j. beth.2010.08.006.

Paul, R., Campbell, D., Gilbert, K., \& Tsiouri, I. (2013). Comparing spoken language treatments for minimally verbal preschoolers with autism spectrum disorders. Journal of Autism and Developmental Disorders, 43(2), 418-431. https://doi.org/10.1007/s10803-012-1583-z.

Schlosser, R. W., Sigafoos, J., \& Koul, R. K. (2009). Speech output and speech-generating devices in autism spectrum disorders. In Mirenda, P., Iacono, T. (Eds.), Autism Spectrum Disorders and AAC (pp. 141-169). Paul H. Brookes Publishing Co.

Schlosser, R. W., Sigafoos, J., Luiselli, J. K., Angermeier, K., Harasymowyz, U., Schooley, K., \& Belfiore, P. J. (2007). Effects of synthetic speech output on requesting and natural speech production in children with autism: A Preliminary study. Research in Autism Spectrum Disorders, 1(2), 139-163. https://doi.org/10.1016/j.rasd.2006.10.001.

Schlosser, R. W., \& Wendt, O. (2008). Effects of augmentative and alternative communication intervention on speech production in children with autism: A systematic review. American Journal of Speech-Language Pathology, 17(3), 212-230. https://doi.org/10.1044/1058-0360(2008/021).

Tincani, M., Crozier, S., \& Alazetta, L. (2006). The picture exchange communication system: Effects on manding and speech development for school-aged children with ASD. Education and Training in Developmental Disabilities, 41(2), 177-184.

Torelli, J. N., Lambert, J. M., Da Fonte, M. A., Denham, K. N., Jedrzynski, T. M., \& Houchins-Juarez, N. J. (2016). Assessing acquisition of and preference for mand topographies during functional communication training. Behavior Analysis in Practice, 9, 165-168. https://doi.org/10.1007/s4061 7-015-0083-y.

Vannest, K. J., \& Ninci, J. (2015). Evaluating intervention effects in single-case research designs. Journal of Counseling \& Development, 93(4), 403-411. https://doi.org/10.1002/jcad.12038.

Wodka, E. L., Mathy, P., \& Kalb, L. (2013). Predictors of phrase and fluent speech in children with ASD and severe language delays. American Academy of Pediatrics, 131(4), 1128-1134. https://doi. org/10.1542/peds.2012-2221.

Publisher's Note Springer Nature remains neutral with regard to jurisdictional claims in published maps and institutional affiliations. 